distance of $\approx 1500$ nucleotides, indicating that overlap with the promoter site is most likely. A common sequence motif around these CpG sites was not immediately detectable but requires more detailed analysis.

Conclusions Microarray based comparative analysis of transcriptional and epigenetic differences suggests a detailed picture of methylation associated gene regulation and enables to generate an epigenetic map of relevant CpG site for genes expressed and regulated in immune cell types. As many of the microarray based suspected $\mathrm{CpG}$ sites of a defined gene did not match with differential gene expression, epigenetic profiling with microarrays has to be interpreted carefully.

\section{A7.6 COMPARATIVE TRANSCRIPTOME ANALYSIS OF HUMAN AND MOUSE SYNOVIAL FIBROBLAST RESPONSES TO TNF}

doi:10.1136/annrheumdis-2013-203221.6

'Thomas Häupl, 'Till Sörensen, 'Bijana Smiljanovic, 'Marc Bonin, ${ }^{2}$ Andreas Grützkau, ${ }^{3}$ Christoforos Nikolaou, ${ }^{3}$ loannis Pandis, ${ }^{3}$ George Kollias, ${ }^{4}$ Anthony Rowe. ${ }^{1}$ Dept. of Rheumatology and Clinical Immunology, Charité University Medicine, Berlin; ${ }^{2}$ German Arthritis Research Center, Berlin; ${ }^{3}$ Biomedical Sciences Research Centre Alexander Fleming', Institute of Immunology, Vari; ${ }^{4}$ Janssen Research and Development

Background and Objectives Animal models are essential for development of new therapeutics to test their effectiveness and to control for side effects. Targeted therapies with biologics reveal differences in responsiveness and suggest substantial differences in the molecular pathomechanisms of human as well as murine arthritis. In order to characterise such differences, we started a pilot investigation based on published transcriptomes to develop bioinformatic strategies of interspecies gene expression comparison.

Materials and Methods A GEO repository search for compatible data sets on arthritis in human and mouse revealed the Affymetrix based transcriptomes of synovial fibroblasts from rheumatoid arthritis (RA) and osteoarthritis (OA) patients (GEO-accession: GSE13837) and mouse BALBc wt (GSE17160) with similar stimulation (TNF-alpha) and time point after stimulation (12 hours). MAS5 algorithms of signal extraction and pairwise comparison were performed. The BioRetis database was used for group comparisons, candidate gene selection and data sharing. Differentially expressed genes were exported for interspecies comparison in a filemaker database. Affymetrix annotation files were used for mapping of orthologous genes.

Results The number of differentially expressed genes induced by TNF in synovial fibroblast from RA, OA and BALBc were 1584, 1476 and 503 respectively. Comparison of genes overexpressed in RA and OA revealed up to $70 \%$ (1034 genes) overlap. Murine and human response was similar for 197 genes in all three groups, 45 only between BALBc and RA and 24 only between BALBc and OA. Ranking the genes by dominance in change call and/or fold change, 7 genes were overlapping between the top 10 of both human cells, $\mathrm{OA}$ and RA, and 13 out of the top 20. All overlapping genes in human revealed a high correlation $(R=0.795)$. In contrast, overlap between RA and BALBc SFbl responses revealed only 4 genes out of the top 10 and 5 out of the top 20 in each and a weaker correlation (0.392). Pathway tools including IPA may identify TNF as one potential upstream regulator. However, the reference list of expected gene candidates for scoring in IPA seemed to be about 2.5 fold longer in human and to include less than $60 \%$ of the murine reference candidates.

Conclusions Interspecies comparison in this defined experiment revealed first insight in common responses but also demonstrates that differences can be observed even in most dominant candidate genes. Therefore, systematic investigations are needed to provide a reliable basis for comparative mapping of the molecular pathomechanisms in mouse models and human disease.

Acknowledgement BTCure IMI grant agreement no. 115142 A7.7 DIFFERENT GENETIC BACKGROUND OF DERMATOMYOSITIS
AND POLYMYOSITIS IN A SINGLE CENTRE COHORT

doi:10.1136/annrheumdis-2013-203221.7

'M Remakova, 'M Skoda, 'T Svitalkova, 'M Faustova, 'L Plestilova, ${ }^{2} \mathrm{Z}$ Betteridge, ${ }^{1} \mathrm{H}$ Mann, ${ }^{1} \mathrm{~J}$ Vencovsky, ${ }^{1} \mathrm{O}$ Krystukova, ${ }^{1} \mathrm{P}$ Novota. I' Institute of Rheumatology, Prague, Czech Republic; ${ }^{2}$ Royal National Hospital for Rheumatic Diseases, Bath, UK

Background and Objectives The idiopathic inflammatory myopathies (IIM) are systemic connective tissue diseases in which autoimmune pathology is responsible for promotion of chronic muscle inflammation and weakness. As in many other autoimmune diseases, the development of IIM is also associated with genes of HLA complex. The aim of this study was to determine the basic relation between alleles of HLA genes and IIM.

Materials and Methods We have performed low to high resolution genotyping to characterise the allelic profiles of HLA-DRB1, -DOB1 and -DOA1 loci in a large group of single centre cohort of patients suffering from IIM $(n=269)$. The genomic DNA was prepared by standard DNA extraction methods and the HLA typing was done using the commercial LABType ${ }^{\circledR}$ SSO kit (One Lambda, USA). Statistical evaluation of results was done with chi-2 test and Fisher exact test. Autoantibody profiles were analysed with radioactive immunoprecipitation

Results The frequencies of HLA-DRB1*03:01 and -DRB1*16:01 alleles were increased in IIM patients and the difference reached statistical significance when compared to healthy controls $(\mathrm{P}<0.01$ for DRB1*03:01; P < 0.05 for DRB1*16:01). Different alleles were associated with dermatomyositis (DM) or polymyositis (PM). The frequency of DRB1*16:01 was significantly higher in DM patients $(\mathrm{P}<0.01)$, whereas the frequency of DRB1*03:01 was higher in patients suffering from PM $(\mathrm{P}<0.01)$, when compared to controls.

Presence of HLA-DRB1*03:01 allele was associated with antiJo-1, anti-Ro52, or anti-Pm-Scl positivity in all IIM patients $(\mathrm{P}<0.05)$. Interestingly, the DRB1*03:01 allele was also associated with the negativity for anti-p155/140 in our patients $(\mathrm{P}<0.01)$.

The DRB1*16:01 allele was associated with negativity of all studied autoantibodies, particularly in subgroup of DM patients $(\mathrm{P}<0.05)$.

Conclusions This study identifies different genetic background between patients with dermatomyositis and polymyositis in a homogenous population of patients from a single centre.

Acknowledgement This work is supported by Internal Grant Agency of Ministry of Health of the Czech Republic NT/12438-4 and NT/13699.

\section{A7.8 DNA DEMETHYLATION IN SALIVARY GLAND EPITHELIAL CELLS FROM PATIENTS WITH PRIMARY SJÖGREN'S SYNDROME MAY BE ASCRIBED TO INFILTRATING B CELLS}

doi:10.1136/annrheumdis-2013-203221.8

${ }^{1,2}{ }^{2}$ ves Renaudineau, ${ }^{1,3} Y_{0}$ sra Thabet, ${ }^{1}$ Christelle Le Dantec, ${ }^{3} \mathrm{lbtissem}$ Ghedira, ${ }^{1,4} \mathrm{~V}$ alérie Devauchelle, ${ }^{1,4}$ Divi Cornec, ${ }^{1}$ Jacques-Olivier Pers. ${ }^{1}$ Research Unit EA2216 "Immunology, Pathology and Immunotherapy", European University of Brittany, Brest University Medical School Hospital, Brest, France; 'Laboratory of Immunology and Immunotherapy, CHRU Morvan, Brest, France; "Research Unit 03UR/07-02 "Autoimmunity and Allergy", Faculty of Pharmacy, Monastir, Tunisia; " ${ }^{\text {Unit }}$ of Rheumatology, CHRU Cavale Blanche, Brest, France

Background and Objectives Sjögren's syndrome (SS) is an autoimmune exocrinopathy characterised by an epithelium injury surrounded by dense lymphocytic infiltrates composed of activated $\mathrm{T}$ and $\mathrm{B}$ cells. Present at the interface of genetic and environmental risk factors, epigenetic modifications are suspected to play a key role in SS. Accordingly, we decided to further characterise DNA methylation in SS. 